\title{
Fermionic Chern insulator from twisted light with linear polarization
}

\author{
Utso Bhattacharya $\odot,{ }^{1,2, *}$ Swati Chaudhary, ${ }^{3,4,5,6}$ Tobias Grass $\odot,{ }^{1}$ Allan S. Johnson $\odot,{ }^{1}$ \\ Simon Wall $\odot,{ }^{1,7}$ and Maciej Lewenstein ${ }^{1,8}$ \\ ${ }^{1}$ ICFO-Institut de Ciencies Fotoniques, The Barcelona Institute of Science and Technology, \\ Av. Carl Friedrich Gauss 3, 08860 Castelldefels (Barcelona), Spain \\ ${ }^{2}$ Max-Planck-Institut für Quantenoptik, D-85748 Garching, Germany \\ ${ }^{3}$ Institute of Quantum Information and Matter and Department of Physics, \\ California Institute of Technology, Pasadena, California 91125, USA \\ ${ }^{4}$ Department of Physics, The University of Texas at Austin, Austin, Texas 78712, USA \\ ${ }^{5}$ Department of Physics, Northeastern University, Boston, Massachusetts 02115, USA \\ ${ }^{6}$ Department of Physics, Massachusetts Institute of Technology, Cambridge, Massachusetts 02139, USA \\ ${ }^{7}$ Department of Physics and Astronomy, Aarhus University, Ny Munkegade 120, 8000 Aarhus C, Denmark \\ ${ }^{8}$ ICREA, Pg. Lluis Companys 23, 08010 Barcelona, Spain
}

(Received 29 September 2020; revised 16 November 2021; accepted 31 January 2022; published 9 February 2022)

\begin{abstract}
The breaking of time-reversal symmetry is a crucial ingredient to topological bands. It can occur intrinsically in materials with magnetic order, or be induced by external fields, such as magnetic fields in quantum Hall systems or circularly polarized light fields in Floquet Chern insulators. Apart from polarization, photons can carry another degree of freedom, orbital angular momentum, through which time-reversal symmetry can be broken. In this Letter we pose the question of whether this property allows for inducing topological bands via a linearly polarized but twisted light beam. To this end we study a graphenelike model of electrons on a honeycomb lattice interacting with a twisted light field. To identify the topological behavior of the electrons, we calculate their local markers of Chern number and monitor the presence of in-gap edge states. Our results are shown to be fully analogous to the behavior found in paradigmatic models for static and driven Chern insulators, and realizing the state is experimentally straightforward. With this, our work establishes a mechanism for generating fermionic topological phases of matter that can harness the central phase singularity of an optical vortex beam.
\end{abstract}

DOI: 10.1103/PhysRevB.105.L081406

In addition to spin angular momentum (SAM), light can also carry orbital angular momentum (OAM) [1,2], giving rise to a huge variety of novel opportunities: OAM transfer via light-matter interactions can allow dipole-forbidden atomic transitions, as experimentally demonstrated for a system of trapped ions [3] and theoretically shown for semiconducting and semimetallic materials [4,5]. It can also be used to generate topological defects in atomic [6] or excitonic [7] condensates, and OAM beams have already found new applications [8] in a variety of fields which include optical communication [9,10], quantum information [11], cosmology [12], and attophysics [13,14]. Despite these advances, the possibility of impinging these beams to create fermionic topological phases of matter has not yet been explored.

A paradigmatic model for topological matter was first proposed by Haldane [15]. The Haldane model considers electrons on a graphenelike lattice, and by incorporating staggered magnetic fluxes threading through every plaquette of the lattice, the model achieves breaking of the time-reversal symmetry (TRS), while translational invariance remains intact and nearest-neighbor hoppings do not pick up any magnetic flux. The second-nearest-neighbor hoppings, however, pick

*utso.bhattacharya@icfo.eu up a net flux which is opposite for the two sublattices. As can be inferred from the Chern number which becomes \pm 1 , these complex hoppings of second-neighbor electrons create a topological Chern insulator. With this the Haldane model has provided the conceptual mechanism for theoretical and experimental research exploring various topologically distinct phases of matter. The tremendous importance of this scheme lies in the fact that now the (anomalous) quantum Hall effect can appear as an intrinsic property of a band structure, rather than being caused by an external magnetic field. In the past few years, the challenging physical realization of the Haldane model has been achieved in different systems, including magnetically ordered topological insulators [16] and hexagonal arrays of helical waveguides [17]. In addition to these examples where time-reversal symmetry is broken by intrinsic properties of the real or synthetic material, there have also been realizations with cold atoms in graphenelike optical lattices [18] where time-reversal symmetry is broken through circular lattice shaking, or more recently in a real graphene lattice [19] by driving the system with circularly polarized light.

Such a realization of the Haldane model using Floquet engineering was first proposed by Oka and Aoki [20]. They showed that irradiating a monolayer of graphene with circularly polarized $(\mathrm{CP})$ light can result in the creation of 
a Floquet-induced Haldane mass. The spin polarization of the light introduces a chirality or a handedness by breaking the TRS of the two-band system. The two eigenstates of the Floquet Hamiltonian then acquire nonzero Chern numbers which in turn leads to a nontrivial band topology. The spin polarization or the spin angular momentum of $\mathrm{CP}$ light being odd under TRS is in fact responsible for the generation of a Haldane mass in the Floquet system. The CP light used there carries SAM but no net OAM.

In this Letter we address, and answer affirmatively, the question of whether, instead of using CP light with zero net OAM, it is possible to use linearly polarized light (zero SAM), but with some integer OAM, to create a Floquet Chern insulator. Considering the vast applicability of OAM light and the fact that Haldane model serves as a building block for many other topological phases, our work provides a major stepping stone to harness their combined potential through Floquet engineering.

We begin with the tight-binding model of graphene. It consists of a monolayer of hexagonal lattice of carbon atoms with two $\pi$ bands [21]. Its Hamiltonian $H_{0}$ is well modeled by nearest-neighbor hopping of electrons between two sublattices $\mathrm{A}$ and $\mathrm{B}$ :

$$
\begin{aligned}
H_{0}= & -t_{0} \sum_{\mathbf{r}_{i}, j=1,2,3}\left(b_{\mathbf{r}_{i}+\mathbf{d}_{j}}^{\dagger} a_{\mathbf{r}_{i}}+a_{\mathbf{r}_{i}}^{\dagger} b_{\mathbf{r}_{i}+\mathbf{d}_{j}}\right) \\
& +\Delta_{0} \sum_{\mathbf{r}_{i}}\left(a_{\mathbf{r}_{i}}^{\dagger} a_{\mathbf{r}_{i}}-b_{\mathbf{r}_{i}+\mathbf{d}_{1}}^{\dagger} b_{\mathbf{r}_{i}+\mathbf{d}_{1}}\right) .
\end{aligned}
$$

The fermionic operators $c_{\mathbf{r}}\left(c_{\mathbf{r}}^{\dagger}\right)$ annihilate (create) an electron at position $\mathbf{r}$ with $c=a, b$ for sublattices $\mathrm{A}$ and $\mathrm{B}$, respectively. The vectors $\mathbf{r}_{i}$ span sublattice $\mathrm{A}$, and $\mathbf{d}_{i}$ are the position vectors to the three nearest neighbors around each A site of the hexagonal plaquette [22]. The first term in $H_{0}$ describes the hopping of electrons between the two sublattices A and B with tunneling strength $t_{0}$, and the second term accounts for a staggered sublattice symmetry-breaking mass $\Delta_{0}$, where the sublattice symmetry breaking can occur in a number of ways [23-26].

Under periodic boundary conditions the system is translationally invariant, and for $\Delta_{0}=0$ it has a gapless spectrum at some isolated points in the quasimomentum space, the quasimomentum being a good quantum number due to translational invariance. Such points are called Dirac points (DP) due to their massless relativistic dispersion at very low energies. These gapless points are protected by three symmetries: chiral (same mass of the two sublattices), time-reversal, and crystal symmetry $C_{3}$. However, placing the monolayer on a substrate such as hexagonal silicon carbide or hexagonal boron nitride introduces an energy difference between the sublattices [27], described by the Semenoff mass term [28], which breaks the sublattice or chiral symmetry. Nonetheless, such finite value of $\Delta_{0}$ only results in a gap that is topologically trivial, due to the presence of TRS. The trivial topology is evidenced by the zero value of a topological invariant called the Chern number calculated over the filled bands of the system. Therefore, to realize a topologically nontrivial insulator, TRS must be broken.

We now consider two situations where we irradiate a graphene monolayer with (a) a circularly polarized light with zero OAM and (b) a linearly polarized vortex beam with finite OAM. We only consider the nearest-neighbor terms for the driven Hamiltonians because the next-nearest-neighbor term is an order of magnitude smaller than the dominant nearest-neighbor interaction. In this work we compare these two situations and demonstrate that even the linearly polarized OAM beam can make the system topologically nontrivial. Here we use the velocity gauge to incorporate the effects of the two periodic drives. The electric field of light $\mathbf{E}(t)$ in the Coulomb gauge is related to the vector potential via the relation $\mathbf{E}(t)=-\frac{\partial \mathbf{A}(t)}{\partial t}$; we also set $\hbar=1$ and speed of light $c=1$. We consider the effect of this vector potential on the hopping term by making the Peierls substitution [22]. To analyze the two cases mentioned above, we employ Floquet theory and use van Vleck high-frequency expansion [29] to derive an effective Floquet Hamiltonian. Numerically, using exact diagonalization as well as a semianalytic construction, we study the Floquet Hamiltonian in both situations.

We first discuss the case of graphene, which is taken to be placed in the $x-y$ plane and irradiated with CP light at normal incidence. The $\mathrm{CP}$ light is characterized by the vector potential:

$$
\mathbf{A}=A_{0} \cos \omega t \hat{x} \pm A_{0} \sin \omega t \hat{y},
$$

where $\hat{x}, \hat{y}$ are orthonormal unit vectors in Cartesian coordinates, \pm stands for right- or left-circular polarization of the light field, $\omega$ is the frequency, and $A_{0}$ is the amplitude of the vector potential which in the dipole approximation is considered spatially uniform over the whole lattice. It can be shown that this drive generates complex next-nearestneighbor (NNN) hopping terms with opposite sign for $A$ and $B$ sites. As a result of this term, the system becomes equivalent to the static Haldane Chern insulator. In this case the NNN hopping gives rise to a TRS-breaking mass term $m_{\mathrm{CP}} \propto \frac{t_{0}^{2}\left(J_{1}\left(A_{0}\right)\right)^{2}}{\omega}$ for each valley in the reciprocal space, where $J_{1}$ denotes the first Bessel function. This mass term competes with the Semenoff mass term to push the system to a topological phase with nonzero Chern invariant. For large frequencies and small electric field amplitudes $E_{\text {amp }}=i \omega A_{0}$, this terms goes as $\frac{E_{\text {amp }}^{2}}{\omega^{3}}$, see [22].

We now turn our attention to the second scenario, where we shine a linearly polarized OAM beam on graphene (see Fig. 1 in [22]). The lattice vectors of graphene in our case are given by $\mathbf{a}_{1}=a(\sqrt{3} / 2,3 / 2), \mathbf{a}_{2}=a(\sqrt{3} / 2,-3 / 2)$, whereas the nearest-neighbor displacement vectors assume the values $\mathbf{d}_{1}=a(0,1), \mathbf{d}_{2}=a(\sqrt{3} / 2,-1 / 2), \mathbf{d}_{3}=$ $a(-\sqrt{3} / 2,-1 / 2) \ldots$. For the OAM drive we use a vector potential given by

$$
\mathbf{A}(x, y)=\left[A_{x}(r) e^{i l \phi} e^{i \omega t}+A_{x}^{*}(r) e^{-i l \phi} e^{-i \omega t}\right] \hat{x},
$$

where $l$ is the integer quantized OAM, which we set to be \pm 1 here; $\phi=\tan ^{-1}\left(\frac{y-y_{0}}{x-x_{0}}\right)$ and in the present case, we chose its center at $\left(x_{0}, y_{0}\right)=(0,0)$ so that it does not lie directly on any lattice site. We immediately note that the vector potential is position dependent and thus breaks translational invariance of the lattice. As a result of this spatial dependence, quasimomenta $k_{x}, k_{y}$ are no longer good quantum numbers. Therefore to study the system a real-space analysis is imperative. The 

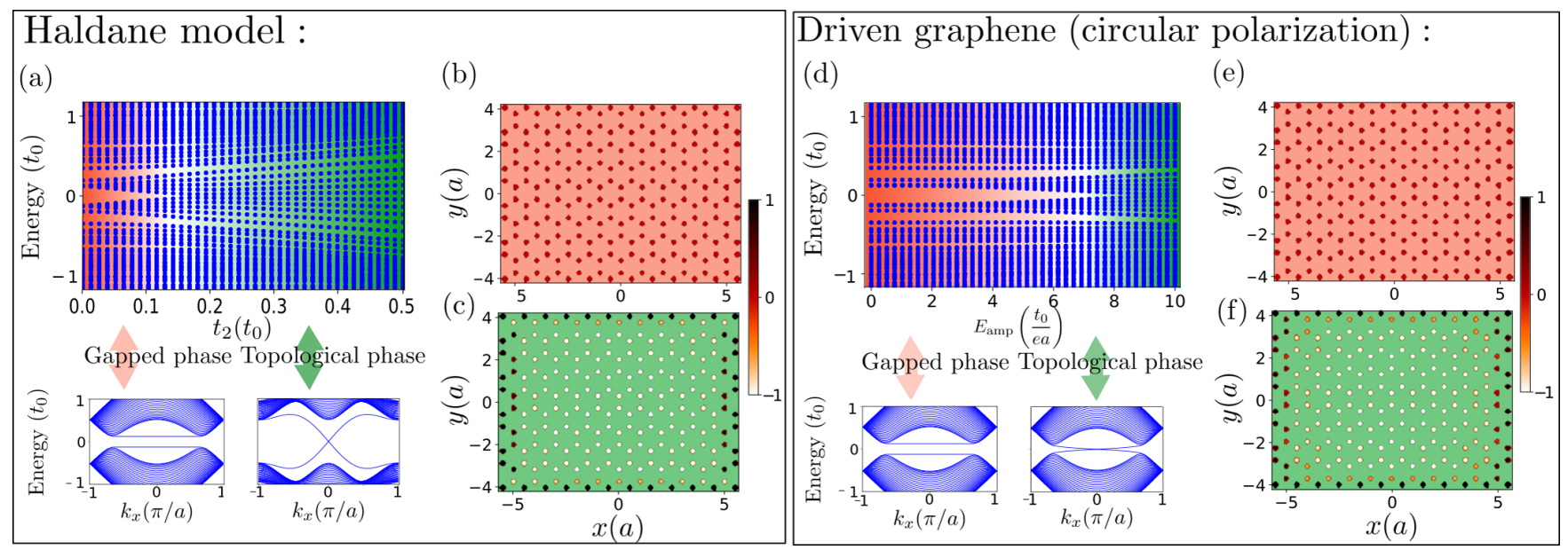

FIG. 1. Static vs driven Chern insulator. (a) Spectra for Haldane model $\left(\Delta_{0}=0.125 t_{0}\right)$ : Energy vs next-nearest-neighbor hopping $t_{2}$ in a system with open boundary (upper plot) and energy vs wave vector $k_{x}$ in a system with periodic boundary (lower plots), for gapped and topological regimes, respectively. The topological regime is characterized by edge states crossing the band gap. (b) Local markers of Chern number $(\mathrm{LMCN})$ in the trivially gapped regime (graphene Hamiltonian) and (c) LMCN in the nontrivial regime (Haldane Hamiltonian). (d) Floquet spectra for graphene driven by circularly polarized light $\left(\Delta_{0}=0.125 t_{0}, \hbar \omega=15 t_{0}\right)$ : Energy vs driving amplitude $E_{\text {amp }}$ in a system with open boundary (upper plot), and energy vs wave vector $k_{x}$ in a system with periodic boundary (lower plots), for gapped and topological regimes, respectively. (e) $\mathrm{LMCN}$ in the trivial regime, and (f) $\mathrm{LMCN}$ in nontrivial regime, of driven graphene.

amplitude $A_{x}(r)$ is a function of radial position $r$ with a singularity at $r=0$, as, for instance, in a Laguerre-Gaussian or a Bessel beam, but for now we choose a constant amplitude $A_{0}$ for $r \neq 0$ so that we can solely focus on the phase of the light which generates the OAM. Again, we include the vector potential via Peierls substitution to derive a Floquet Hamiltonian [22]. However, in contrast to the CP drive, in this case both the NNN hopping amplitude and phase in the effective Hamiltonian are position dependent.

Generally, a direct measure of the bulk topology of a system is the Chern invariant, which is usually defined as an integral over the Berry curvature of a band and thus requires the presence of translational invariance. Since the present scenario lacks translational invariance, we cannot calculate the quasimomentum-space bulk Chern number, and hence we rely on the real-space formulation discussed in Refs. [30,31]. In this formalism, one can infer the Chern number of the system from the macroscopic average of a gauge-invariant microscopic function known as local marker of Chern number (see [22]). We quickly present the result of the topological invariant from the local markers of Chern number (LMCN) for the following three situations: (i) trivially gapped graphene, (ii) Haldane model, (iii) CP-driven graphene (nonzero SAM, zero OAM), and then compare the results with that of (iv) OAM-driven graphene (zero SAM, nonzero OAM). For all cases we consider a lattice under open boundary condition (OBC) in both directions, and we plot the LMCN for every point in the lattice: Fig. 1(b) shows the result for case (i), with the local Chern number being zero everywhere in the bulk and at the edges. This correctly captures the fact that TRS is intact, and the energy gap due to broken sublattice symmetry leaves the system topologically trivial. In contrast, Figs. 1(c) and 1(f), which correspond to cases (ii) and (iii) of broken TRS, clearly demonstrate topologically nontrivial behavior. Specifically, the LMCN exhibits edge states (in black as shown in the color bars alongside the figure) with averaged bulk Chern number +1 . We also note that the edge $\mathrm{LMCN}$ and the averaged bulk Chern number have equal and opposite contributions, as expected according to Ref. [30]. Furthermore, on reversing the sign of the mass term in the static case or the polarization of light in the driven scenario, the edge (and bulk) value of LMCN reverses as expected (see [22]). These results establish that LMCN are an excellent means of identifying topological phases in the absence of translational invariance. All numerical simulations were performed using Kwant [32].

We are now set to apply this tool to case (iv), our prime case of interest. Figure 2(b) shows the corresponding LMCN plot for a rectangular system, while Fig. 2(c) shows the LMCN for a sample with Corbino geometry. In the rectangular system we have assumed that the light beam is of constant intensity, i.e., $A_{x}(r)=$ const. in Eq. (3). Indeed, the spatial phase twist of the beam is enough to obtain a Chern insulating phase (see [22]). In practice, however, the phase twist stems from a phase singularity which requires that the intensity of the light vanishes in one point. We have taken this into account by also considering a Bessel beam, $A_{x}(r) \sim J_{l}(q r)$. For the system to be in a Chern phase, the intensity should be nonzero beyond a critical value, depending upon its frequency and also the Semenoff (nontopological) mass. Therefore we have considered the case where the optical vortex of a Bessel beam is placed within the annular hole of a Corbino disk, albeit making sure that the intensity from the beam reaches its peak value between the two edges of the sample. As further discussed below, this can be achieved either by making the optical vortex narrow (controlled by the wave vector $q$ ) or by using large enough samples. The latter option includes the scenario where the annular hole extends over more unit cells than shown in Fig. 2(c), as well as the case of artificial graphene systems [33] with a potentially much larger lattice constant (on the order of $100 \mathrm{~nm}$ ).

Both plots, Figs. 2(b) and 2(c), conclusively reveal that the system hosts chiral topological edge states. In case of the 

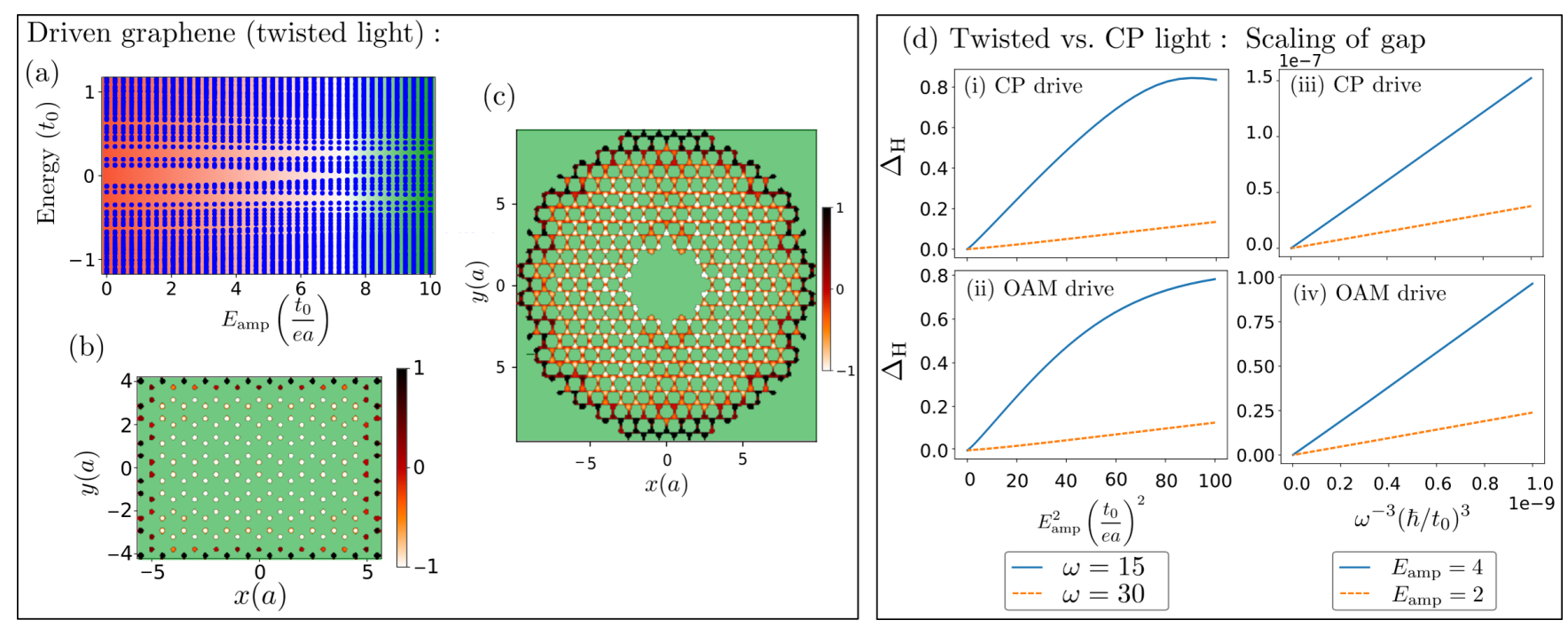

FIG. 2. Chern insulator due to OAM drive. (a) Floquet spectrum: Energy vs driving amplitude $E_{\text {amp }}$ in a system with open boundary $\left(\Delta_{0}=0.125 t_{0}, \hbar \omega=15 t_{0}\right)$ : Closing of the band gap due to strong driving indicates the transition into a nontrivial phase. (b, c) Local Chern numbers: Topologically nontrivial behavior of OAM-driven graphene is evident from the nonzero local Chern numbers in a rectangular sample (b) and a corbino-shaped geometry (c). (d) Scaling of the Haldane-like gap, $\Delta_{H}$, with driving amplitude and frequency for both CP-driven and OAM-driven graphene. Here $\Delta_{H}=\frac{2 \Delta_{0}-E_{\text {gap }}}{2 \Delta_{0}}$, where $E_{\text {gap }}$ is the band gap shown in (a).

Corbino sample, the inner circular edge of the sample has a nonzero integer quantized $\mathrm{LMCN}$ with value -1 (white), whereas the outer edge of the sample has an LMCN with value +1 (black). Similarly, in case of the rectangular system, the LMCN at the sample edge averages to +1 (black). Furthermore, in both cases the local Chern number changes sign along the two edges on reversing the OAM of the beam, indicating that the chirality of these states is due to the OAM of the beam. We have only shown results for \pm 1 here. But we have verified that any nonzero OAM can produce a Chern insulating phase (see results for $l=+2$ in Fig. 5 in Ref. [22]). The sign of the Chern number depends on the sign of the OAM, so it is possible to flip between a positive and a negative Chern phase by reversing the OAM direction. This conclusion is also corroborated by the constant intensity case (rectangular sample), which reveals that the phase winding of the beam is sufficient to generate the Chern insulating behavior. We now want to take a deeper look at the topological edge states in the three cases (ii)-(iv) discussed above. To this end, under complete $\mathrm{OBC}$ we plot the energy spectra against the parameter which controls the complex NNN hopping. In the static Haldane model, this is simply $t_{2}$, whereas for the driven cases it is the amplitude $E_{\mathrm{amp}}$ of the light field. All spectra shown in Figs. 1(a), 1(d), and 2(a) have one feature in common: the energy gap due to a finite Semenoff mass closes when the control parameter becomes sufficiently strong, which distinguishes a gapped phase (shaded in red) from a gapless (at edge) phase (shaded in green). The observed gap closing is due to the occurrence of an in-gap state, which can easily be visualized for case (ii) and case (iii). In these cases the presence of translational symmetry allows us to apply PBC along the $x$ direction, and the lower plots in Figs. 1(a) and 1(d) show the resulting energy dispersion in both the gapped and the gapless regime. We observe that the vanishing of the gap is due to a single energy level which connects one valley of the conduction band to the opposite in the valence band. In contrast, in the gapped regime the energy dispersion exhibits topologically trivial edge states which connect the Dirac modes within each band but without crossing the gap between conduction and valence band. Due to the lack of translational invariance, an analogous plot for the quasienergy spectra against a conserved quasimomentum is not possible in the OAM-driven scenario, but the appearance of in-gap states is seen from Fig. 2(a) and hints for a nontrivial topological phase with chiral edge states. The presence of chiral edge states with quantized conductance can also be observed through transport measurements, as has been confirmed in this case by the calculation of the edge conductance (see "Edge Conductance" section in Ref. [22]).

We finally want to reveal the order of the amplitude and frequency of OAM light required to produce such a Floquet Chern insulator. A semianalytical approach is adopted in which we construct an effective Floquet Hamiltonian once again via a van Vleck transformation, but this time in the limit where only the leading-order term is retained (see [22]), that is, the term which scales with $E_{\text {amp }}^{2} / \omega^{3}$, or the singlephoton sector. We use numerical diagonalization to confirm that the quasienergy spectrum around zero energy for this Hamiltonian and with that obtained from exact numerical approach (see Fig. 2 in [22]) match at high frequencies and small amplitudes of the light field. Most importantly, the Floquet Chern insulator from OAM possesses a mass gap, $\Delta_{H}$, which scales as $E_{\text {amp }}^{2} / \omega^{3}$, and thus it exhibits exactly the same quantitative behavior as the CP light-driven system [as shown in Fig. 2(d)]. The identical topological behavior of the eigenstates captured through the LMCN and the correspondence of the zero energy spectrum is suggestive of a topological equivalence between the effective Hamiltonian and the Hamiltonian of the static Haldane model, which are formally very similar, albeit with position-dependent 
phases along the NNN electron hoppings in the effective Hamiltonian.

Experimentally generating such OAM beams with the high field strengths required to observe the Chern insulator behavior is now routine, both in the laboratory (UV-THz) and at facilities (XUV-x ray). Realizing the geometry simulated here, with uniform intensity outside of the central singularity located in a central aperture, is less standard but not significantly more challenging. The intensity FWHM of the central minimum in OAM beams can be as low as 0.3 wavelengths [34], and with appropriate phase masking the intensity can be made approximately uniform outside of this region without affecting the carried OAM. For high-intensity $x$ rays at $\mathrm{x}$-ray free electron lasers this is already sufficient to address graphene in the geometry shown in Fig. 2(c), which is limited to a radial size of ten lattice constants. Longer wavelengths require larger samples or, alternatively, larger lattice constants. Artificial forms of graphene can have lattice constants up to $100 \mathrm{~nm}$ [33]. In all cases the topologically nontrivial state can be detected via the emergence of an anomalous Hall current as in Ref. [19]. Since the gap is found to saturate with high field strengths [Fig. 2(d)], it should also be possible to decrease the effective width of the minimum by going to higher field strengths, analogously to stimulation emission depletion (STED) microscopy [35], allowing optical fields to produce the effect directly in graphene.

Circularly polarized light pulses can induce the Floquet Chern phase in monolayer graphene [19]. In this Letter we have demonstrated that, amazingly, the Floquet Chern phase can also be induced via linearly polarized light with nonzero OAM. To this aim we calculated the local markers of Chern number and monitored the presence of in-gap edge states. This opens new paths for the light-induced generation of topological states in solid-state systems consisting of monolayers, bilayers, etc. It would be interesting to carry over these ideas to quantum simulator platforms such as cold atoms. Another interesting possibility could be to combine OAM and CP to create and impinge on the substrate even more "exotic" topological light pulses, such as the ones carrying topological knots [36,37], or bichromatic fields which can be used, for instance, to generate XUV harmonics with a self-torque, i.e., time-dependent OAM [38].

Moreover, the OAM of light is a degree of freedom that can be tuned independently of the circular spin polarization to induce a topological phase. More importantly, in artificial platforms such as optical lattices, it may be possible to create multilevel topological models based upon the excited states with higher OAM that can be reached by shining OAM light. It is more like synthetic degrees of freedom that circularly spin-polarized light cannot access. OAM light can also now be used in conjugation with the $\mathrm{CP}$ degree of freedom. For instance, it should be possible to modify the Dirac spectrum of the system, to move around the Dirac points, and to merge them to open a gap or generate more Dirac points using the circular spin polarization, while the OAM degree of freedom could simultaneously be used to induce interesting topological phases in such systems, even phases with large Chern numbers. It can also find valleytronic applications in graphenelike systems where a topological mass gap could be induced by the OAM, while the handedness of the circular polarization can independently and selectively tune the valley pseudospin.

We thank Gil Refael for enlightening discussions. U.B., T.G., and M.L. acknowledge the ERC AdG NOQIA, Agencia Estatal de Investigación ("Severo Ochoa" Center of Excellence CEX2019-000910-S, Plan National FIDEUA PID2019-106901GB-I00/10.13039 / 501100011033, FPI, QUANTERA MAQS PCI2019-1118282 / 10.13039/501100011033), Spanish Ministry MINECO (National Plan 15, Grant FISICATEAMO No. FIS201679508-P, SEVERO OCHOA No. SEV-2015-0522, FPI), European Social Fund, Fundació Cellex, Fundació Mir-Puig, Generalitat de Catalunya (AGAUR Grant No. 2017SGR 1341, CERCA program, QuantumCAT U16-011424, cofunded by ERDF Operational Program of Catalonia 2014-2020), EU Horizon 2020 FET-OPEN OPTOLogic (Grant No. 899794), and the National Science Centre, Poland-Symfonia Grant No. 2016/20/W/ST4/00314, Marie Skłodowska-Curie Grant STREDCH No. 101029393, "La Caixa" Junior Leaders fellowships (ID100010434), and EU Horizon 2020 under Marie Skłodowska-Curie Grant Agreement No. 847648 (LCF/BQ/PI19/11690013, LCF/BQ/PI20/11760031, LCF/BQ/PR20/11770012). S.C. acknowledges support from the Institute of Quantum Information and Matter, an NSF Frontier Center funded by the Gordon and Betty Moore Foundation. T.G. acknowledges financial support from a fellowship granted by the Caixa Foundation (ID100010434, Fellowship Code No. LCF/BQ/PI19/11690013). U.B. acknowledges Cellex-ICFO-MPQ Fellowship funding. A.S.J. acknowledges funding from Marie Skodowska-Curie Grant Agreement No. 754510 (PROBIST).
[1] L. Allen, M. W. Beijersbergen, R. J. C. Spreeuw, and J. P. Woerdman, Orbital angular momentum of light and the transformation of Laguerre-Gaussian laser modes, Phys. Rev. A 45, 8185 (1992).

[2] J. P. Torres and L. Torner, Twisted Photons: Applications of Light with Orbital Angular Momentum (Wiley, New York, 2011).

[3] C. T. Schmiegelow, J. Schulz, H. Kaufmann, T. Ruster, U. G. Poschinger, and F. Schmidt-Kaler, Transfer of optical orbital angular momentum to a bound electron, Nat. Commun. 7, 12998 (2016).
[4] G. F. Quinteiro and P. I. Tamborenea, Twistedlight-induced optical transitions in semiconductors: Free-carrier quantum kinetics, Phys. Rev. B 82, 125207 (2010).

[5] M. B. Farías, G. F. Quinteiro, and P. I. Tamborenea, Photoexcitation of graphene with twisted light, Eur. Phys. J. B 86, 432 (2013).

[6] M. F. Andersen, C. Ryu, P. Cladé, V. Natarajan, A. Vaziri, K. Helmerson, and W. D. Phillips, Quantized Rotation of Atoms from Photons with Orbital Angular Momentum, Phys. Rev. Lett. 97, 170406 (2006). 
[7] M.-S. Kwon, B. Y. Oh, S.-H. Gong, J.-H. Kim, H. K. Kang, S. Kang, J. D. Song, H. Choi, and Y.-H. Cho, Direct Transfer of Light's Orbital Angular Momentum onto a Nonresonantly Excited Polariton Superfluid, Phys. Rev. Lett. 122, 045302 (2019).

[8] Y. Shen, X. Wang, Z. Xie, C. Min, X. Fu, Q. Liu, M. Gong, and $\mathrm{X}$. Yuan, Optical vortices 30 years on: OAM manipulation from topological charge to multiple singularities, Light Sci. Appl. 8, 90 (2019).

[9] J. Wang, J.-Y. Yang, I. M. Fazal, N. Ahmed, Y. Yan, H. Huang, Y. Ren, Y. Yue, S. Dolinar, M. Tur, and A. E. Willner, Terabit free-space data transmission employing orbital angular momentum multiplexing, Nat. Photonics 6, 488 (2012)

[10] N. Bozinovic, Y. Yue, Y. Ren, M. Tur, P. Kristensen, H. Huang, A. E. Willner, and S. Ramachandran, Terabit-Scale Orbital Angular Momentum Mode Division Multiplexing in Fibers, Science 340, 1545 (2013).

[11] A. Nicolas, L. Veissier, L. Giner, E. Giacobino, D. Maxein, and J. Laurat, A quantum memory for orbital angular momentum photonic qubits, Nat. Photonics 8, 234 (2014).

[12] F. Tamburini, B. Thidé, G. Molina-Terriza, and G. Anzolin, Twisting of light around rotating black holes, Nat. Phys. 7, 195 (2011).

[13] F. Kong, C. Zhang, F. Bouchard, Z. Li, G. G. Brown, D. H. Ko, T. J. Hammond, L. Arissian, R. W. Boyd, E. Karimi, and P. B. Corkum, Controlling the orbital angular momentum of high harmonic vortices, Nat. Commun. 8, 14970 (2017).

[14] D. Gauthier, P. R. Ribič, G. Adhikary, A. Camper, C. Chappuis, R. Cucini, L. F. DiMauro, G. Dovillaire, F. Frassetto, R. Géneaux, P. Miotti, L. Poletto, B. Ressel, C. Spezzani, M. Stupar, T. Ruchon, and G. De Ninno, Tunable orbital angular momentum in high-harmonic generation, Nat. Commun. 8, 14971 (2017).

[15] F. D. M. Haldane, Model for a Quantum Hall Effect without Landau Levels: Condensed-Matter Realization of the "Parity Anomaly," Phys. Rev. Lett. 61, 2015 (1988).

[16] C.-Z. Chang, J. Zhang, X. Feng, J. Shen, Z. Zhang, M. Guo, K. Li, Y. Ou, P. Wei, L.-L. Wang, Z.-Q. Ji, Y. Feng, S. Ji, X. Chen, J. Jia, X. Dai, Z. Fang, S.-C. Zhang, K. He, Y. Wang, et al., Experimental observation of the quantum anomalous Hall effect in a magnetic topological insulator, Science 340, 167 (2013).

[17] M. C. Rechtsman, J. M. Zeuner, Y. Plotnik, Y. Lumer, D. Podolsky, F. Dreisow, S. Nolte, M. Segev, and A. Szameit, Photonic Floquet topological insulators, Nature (London) 496, 196 (2013).

[18] G. Jotzu, M. Messer, R. Desbuquois, M. Lebrat, T. Uehlinger, D. Greif, and T. Esslinger, Experimental realization of the topological Haldane model with ultracold fermions, Nature (London) 515, 237 (2014).

[19] J. W. McIver, B. Schulte, F.-U. Stein, T. Matsuyama, G. Jotzu, G. Meier, and A. Cavalleri, Light-induced anomalous Hall effect in graphene, Nat. Phys. 16, 38 (2020).

[20] T. Oka and H. Aoki, Photovoltaic Hall effect in graphene, Phys. Rev. B 79, 081406(R) (2009).

[21] A. H. Castro Neto, F. Guinea, N. M. R. Peres, K. S. Novoselov, and A. K. Geim, The electronic properties of graphene, Rev. Mod. Phys. 81, 109 (2009).
[22] See Supplemental Material at http://link.aps.org/supplemental/ 10.1103/PhysRevB.105.L081406 which describes the used methods and contains discussion of Refs. [39,40].

[23] V. M. Pereira, A. H. Castro Neto, and N. M. R. Peres, Tightbinding approach to uniaxial strain in graphene, Phys. Rev. B 80, 045401 (2009).

[24] Y.-W. Son, M. L. Cohen, and S. G. Louie, Energy Gaps in Graphene Nanoribbons, Phys. Rev. Lett. 97, 216803 (2006).

[25] P. Sessi, J. R. Guest, M. Bode, and N. P. Guisinger, Patterning graphene at the nanometer scale via hydrogen desorption, Nano Lett. 9, 4343 (2009).

[26] U. R. Singh, M. Prada, V. Strenzke, B. Bosnjak, T. Schmirander, L. Tiemann, and R. H. Blick, Sublattice symmetry breaking and ultralow energy excitations in graphene-on- $h \mathrm{BN}$ heterostructures, Phys. Rev. B 102, 245134 (2020).

[27] S. Y. Zhou, G.-H. Gweon, A. V. Fedorov, P. N. First, W. A. de Heer, D.-H. Lee, F. Guinea, A. H. Castro Neto, and A. Lanzara, Substrate-induced bandgap opening in epitaxial graphene, Nat. Mater. 6, 770 (2007).

[28] G. W. Semenoff, Condensed-Matter Simulation of a ThreeDimensional Anomaly, Phys. Rev. Lett. 53, 2449 (1984).

[29] M. Bukov, L. D’Alessio, and A. Polkovnikov, Universal high-frequency behavior of periodically driven systems: From dynamical stabilization to Floquet engineering, Adv. Phys. 64, 139 (2015).

[30] R. Bianco and R. Resta, Mapping topological order in coordinate space, Phys. Rev. B 84, 241106(R) (2011).

[31] A.-L. He, L.-R. Ding, Y. Zhou, Y.-F. Wang, and C.-D. Gong, Quasicrystalline Chern insulators, Phys. Rev. B 100, 214109 (2019).

[32] C. W. Groth, M. Wimmer, A. R. Akhmerov, and X. Waintal, Kwant: A software package for quantum transport, New J. Phys. 16, 063065 (2014).

[33] M. Polini, F. Guinea, M. Lewenstein, H. C. Manoharan, and V. Pellegrini, Artificial honeycomb lattices for electrons, atoms and photons, Nat. Nanotechnol. 8, 625 (2013).

[34] P. Török and P. R. T. Munro, The use of Gauss-Laguerre vector beams in STED microscopy, Opt. Express 12, 3605 (2004).

[35] T. A. Klar, E. Engel, and S. W. Hell, Breaking Abbe's diffraction resolution limit in fluorescence microscopy with stimulated emission depletion beams of various shapes, Phys. Rev. E 64, 066613 (2001).

[36] E. Pisanty, G. J. Machado, V. Vicuña-Hernández, A. Picón, A. Celi, J. P. Torres, and M. Lewenstein, Knotting fractional-order knots with the polarization state of light, Nat. Photonics 13, 569 (2019).

[37] E. Pisanty, L. Rego, J. SanRoman, A. Picon, K. M. Dorney, H. C. Kapteyn, M. M. Murnane, L. Plaja, M. Lewenstein, and C. Hernandez-Garcia, Conservation of Torus-Knot Angular Momentum in High-Order Harmonic Generation, Phys. Rev. Lett. 122, 203201 (2019).

[38] L. Rego, K. M Dorney, N. J Brooks, Q. L Nguyen, C.-T. Liao, J. San Román, D. E Couch, A. Liu, E. Pisanty, M. Lewenstein, et al., Generation of extreme-ultraviolet beams with time-varying orbital angular momentum, Science 364, 9486 (2019).

[39] A. Eckardt and E. Anisimovas, High-frequency approximation for periodically driven quantum systems from a Floquet-space perspective, New J. Phys. 17, 093039 (2015).

[40] M. V Moskalets, Scattering Matrix Approach to Non-Stationary Quantum Transport (World Scientific, Singapore, 2011). 